\title{
A New Conjugate Gradient Method with Sufficient Descent Property
}

\author{
O.B. Akinduko
}

Department of Mathematical Sciences, Adekunle Ajasin University, Akungba-Akoko, Nigeria e-mail: akindukooluwaseun@gmail.com

\begin{abstract}
In this paper, by linearly combining the numerator and denominator terms of the Dai-Liao (DL) and Bamigbola-Ali-Nwaeze (BAN) conjugate gradient methods (CGMs), a general form of DL-BAN method has been proposed. From this general form, a new hybrid CGM, which was found to possess a sufficient descent property is generated. Numerical experiment was carried out on the new CGM in comparison with four existing CGMs, using some set of large scale unconstrained optimization problems. The result showed a superior performance of new method over majority of the existing methods.
\end{abstract}

\section{Introduction}

No investor wants to go for investment without returns or with high risk; hence the need for decision making. Optimization is central to any problem involving decision making which arises from the fields of Engineering, Economics, Science, etc. It entails choosing the best out of various alternatives (Chong and Zak [3]). A way to handle this kind of problem involves solving an unconstrained optimization problem of the form:

$$
\min f(x), x \in \mathbf{R}^{n}
$$

Problems of the form (1) arise in many theoretical fields because most of the optimization problems can be reduced to an unconstrained optimization problem

Received: December 16, 2020; Accepted: February 3, 2021

2010 Mathematics Subject Classification: 90C30.

Keywords and phrases: conjugate gradient method, line search, sufficient descent property, unconstrained optimization, numerical experiment. 
(Mangasarian [11]). The CGM is a computational scheme for solving the unconstrained minimization problem (1). The first CGM was developed in 1952 by Hestenes and Stiefel as an algorithm for solving algebraic equations, which was later applied to nonlinear unconstrained optimization problems as reported by Fletcher and Reeves in 1964. Since then, the CGM has been an area of active research. Out of the many iterative methods for solving (1), the CGM is very popular due to the simplicity of its analysis, its low memory requirements and its ease of implementation. The iterative scheme is given by:

$$
x_{k+1}=x_{k}+\alpha_{k} d_{k}, \quad k=0,1,2, \ldots
$$

where $x_{k}$ is the $k$ th solution iterate to (1), $\alpha_{k}>0$ denotes the step size, usually obtained by a line search and $d_{k}$, given by

$$
d_{k}= \begin{cases}-g_{k} & \text { if } k=0 \\ -g_{k}+\beta_{k} d_{k-1} & \text { if } k \geq 1\end{cases}
$$

is the search direction, $g_{k}=\nabla f\left(x_{k}\right)$ is the gradient and $\beta_{k}$ is a scalar known as the conjugate update parameter. Different choices of $\beta_{k}$ has resulted in different CGMs. Some well known classical CGMs, developed by Hestenes and Stiefel [9], Fletcher and Reeves [7, Fletcher [8], Dai and Liao [4] and Bamigbola et al. [2] are:

$$
\begin{gathered}
\beta_{k}^{H S}=\frac{g_{k}^{T} y_{k-1}}{d_{k-1}^{T} y_{k-1}}, \beta_{k}^{F R}=\frac{\left\|g_{k}\right\|^{2}}{\left\|g_{k-1}\right\|^{2}}, \beta_{k}^{C D}=\frac{\left\|g_{k}\right\|^{2}}{-d_{k-1}^{T} g_{k-1}}, \\
\beta_{k}^{D L}=\frac{g_{k}^{T}\left(y_{k-1}-t s_{k-1}\right)}{d_{k-1}^{T} y_{k-1}}, \text { and } \beta_{k}^{B A N}=\frac{-g_{k}^{T} y_{k-1}}{g_{k-1}^{T} y_{k-1}} .
\end{gathered}
$$

In the classical methods (4- 5), we have $y_{k-1}=g_{k}-g_{k-1}, t>0, s_{k-1}=x_{k}-x_{k-1}$ and $\|$. \| stands for the Euclidean norm.

To any CGM, the determination of the search direction $d_{k}$ and the step size $\alpha_{k}$ is very important. A careful choice of the line search strategy is needed to obtain a descent direction (Nocedal [13]). Basically, two types of line search are used in computing $\alpha_{k}$, namely the exact and inexact line search rules. By the exact line search, $\alpha_{k}$ is computed such that:

$$
\alpha_{k}=\operatorname{argmin}\left\{f\left(x_{k}+\alpha d_{k}\right) ; \alpha \geq 0\right\}
$$


This approach is expensive in terms of evaluating the function and gradient. The limitation of the exact line search led researchers to the use of inexact line search, where $\alpha_{k}$ is computed numerically by ensuring a reasonable reduction in the value of the objective function at a minimal cost. One of the most popular inexact line search is the StrongWolfe line search given by:

$$
f\left(x_{k}\right)-f\left(x_{k}+\alpha_{k} d_{k}\right) \geq-\delta \alpha_{k} g_{k}^{T} d_{k}
$$

and

$$
\left|g\left(x_{k}+\alpha_{k} d_{k}\right)^{T} d_{k}\right| \leq \sigma\left|g_{k}^{T} d_{k}\right|
$$

with $0 \leq \delta \leq \sigma \leq 1$. It is required that the search direction, $d_{k}$ satisfy:

$$
g_{k}^{T} d_{k}<0, \forall k \geq 0
$$

which guarantees a descent direction of $f(x)$ at $x_{k}$.

A class of CGMs known as hybrid CGMs, which are modifications of the classical CGMs have been proposed by various authors. This is due to the part it plays in achieving better computational performance as well as retaining the strong global convergence of the methods involved ( $\mathrm{Li}$ and Zhao [10]). By taking into consideration the convex combination of the numerators and denominators of the update parameters of Fletcher-Reeves and Hestenes-Stiefel methods, Nazareth [12] proposed a two-parameter family of CGMs. Dai and Liao [4] extended this by adding one more parameter, where their three-parameter family included six standard CGMs. By forming a linear combination of the update parameters of the Dai-Yuan and Hestenes-Stiefel methods and that of Fletcher-Reeves and Polak-Ribiere-Polyak methods, $\mathrm{Xu}$ and Kong [15] proposed two new hybrid CGMs, with the aid of the generalized Wolfe line search. Recently, Osinuga and Olofin [14] presented an extended hybrid CGM which was proved to be globally convergent with Armijo-type line search, while Djordjevic [5] proposed another new CGM by a convex combination of the update parameters of Liu-Storey and Fletcher-Reeves methods. 
Desirous to generate many methods by varying coefficients from a linear combination of the numerator and denominator terms of the update parameters of DL and BAN methods, this paper presents a new CGM.

\section{The New Method}

By linearly combining the update parameters of DL and BAN methods, the following general form of the DL-BAN method is proposed:

$$
\beta_{k}^{D B}=\frac{\lambda_{1} g_{k}^{T} y_{k-1}+\lambda_{2} t g_{k}^{T} S_{k-1}}{\omega_{1} d_{k-1}^{T} y_{k-1}+\omega_{2} g_{k-1}^{T} y_{k-1}},
$$

where $\lambda_{i}, \omega_{i} \in \Re$ and $t>0$. Taking $\lambda_{1}=1, \lambda_{2}=-1, \omega_{1}=1$, and $\omega_{2}=0$, we have $\beta_{k}^{D L}$ and by taking $\lambda_{1}=-1, \lambda_{2}=0, \omega_{1}=0$, and $\omega_{2}=1$, we have $\beta_{k}^{B A N}$. Thus, several new methods can be generated from 10 by varying the values of $\lambda_{i}$ and $\omega_{i}$. Therefore, taking $\lambda_{1}=0, \lambda_{2}=1, \omega_{1}=0$, and $\omega_{2}=1$ and $t=1$ from (10), a new hybrid of DL and BAN methods is proposed in this paper and it is given by:

$$
\beta_{k}^{N M}=\frac{g_{k}^{T} s_{k-1}}{g_{k-1}^{T} y_{k-1}}
$$

The following Algorithm is used to implement the NM CG method

\section{Algorithm 2.1}

Step 1: Choose $\epsilon=10^{-6}$ and $x_{0} \in \Re^{n}$, set $k:=0$

Step 2: Stop if $\left\|g_{k}\right\| \leq \epsilon$

Step 3: Compute $\beta_{k}$ based on (11)

Step 4: Compute $d_{k}$ by (3)

Step 5: Compute $\alpha_{k}$ by (7) and (8)

Step 6: Update a new point by (2)

Step 7: Set $k:=k+1$, and return to step 2 . 


\section{Sufficient Descent Analysis of $\beta_{k}^{N M}$ Method}

The sufficient descent analysis of $\beta_{k}^{N M}$ shall be carried out based on the following lemmas

Lemma 3.1. In the Conjugate Gradient Method,

$$
g_{i}^{T} d_{k}=0, k=i-1
$$

Proof. See Chong and Zak ([3]; Lemma 10.2, pp. 174-176) for the proof.

Lemma 3.2. The new $C G$ method $\beta_{k}^{N M}$ satisfy the sufficient descent condition, i.e.,

$$
g_{k}^{T} d_{k} \leq-c\left\|g_{k}\right\|^{2}, \quad 0<c \leq 1 .
$$

Proof. By (3) and (11),

$$
g_{k}^{T} d_{k}=-g_{k}^{T} g_{k}+\beta_{k}^{N M} g_{k}^{T} d_{k-1}=-g_{k}^{T} g_{k}+\frac{g_{k}^{T} s_{k-1}}{g_{k-1}^{T} y_{k-1}} g_{k}^{T} d_{k-1} .
$$

Let the second term be expressed in the form $u^{T} v \leq \frac{1}{2}\left(\|u\|^{2}+\|v\|^{2}\right)$ by making $u=\frac{2}{\sqrt{5}} g_{k}, v=\frac{\sqrt{5}}{2} \frac{s_{k-1} g_{k}^{T} d_{k-1}}{g_{k-1}^{T} y_{k-1}}$ and applying Lemma 3.1, we have:

$$
\begin{aligned}
g_{k}^{T} d_{k} & \leq-\left\|g_{k}\right\|^{2}+\frac{1}{2}\left[\left\|\frac{2}{\sqrt{5}} g_{k}\right\|^{2}+\left\|\frac{\sqrt{5}}{2} \frac{s_{k-1} g_{k}^{T} d_{k-1}}{g_{k-1}^{T} y_{k-1}}\right\|^{2}\right] \\
& \leq \frac{1}{2}\left[\frac{4}{5}\left\|g_{k}\right\|^{2}+\frac{5}{4} \frac{\left\|s_{k-1}\right\|^{2}\left(g_{k}^{T} d_{k-1}\right)^{2}}{\left(g_{k-1}^{T} y_{k-1}\right)^{2}}\right] \\
& \leq-\left\|g_{k}\right\|^{2}+\frac{2}{5}\left\|g_{k}\right\|^{2} \\
& \leq-\frac{3}{5}\left\|g_{k}\right\|^{2}
\end{aligned}
$$

Therefore, $\beta_{k}^{N M}$ method satisfies 13 with $c=\frac{3}{5}$. 


\section{Numerical Consideration}

In this section, a report of the numerical experiment carried out on the new CGM, using a set of large-scale unconstrained minimization problems, taken from Andrei [1], is presented.

\subsection{Computational details}

A total of 27 unconstrained optimization problems, each of dimensions 5000 and 10000, were solved using the Strong Wolfe line search. The iterations were terminated when $\left\|g_{k}\right\| \leq 10^{-6}$, and a failure declared if this condition was not satisfied after 2000 iterations. The nonlinear conjugate gradient algorithm (CGA) was written in Matlab codes and run on a PC with $2.16 \mathrm{GHz}$ processor, 4GB Ram and Windows 10 operating system.

\subsection{Presentation of numerical results}

The numerical results obtained for the new method in comparison with four existing CGMs are presented in Tables 1-2. In tabulating the numerical results, the following notations were used:

Dim - Dimension; NM- new method; CD - Conjugate Descent method; F - Failed; Itr - Iteration; HS - Hestenes-Stiefel method; DL - Dai-Liao method; BAN - Bamigbola-Ali-Nwaeze method; $\left\|g\left(x^{*}\right)\right\|$ - norm of gradient of the objective function at iteration $x^{*}$; $\mathbf{C p u}$ computational time.

The performance profile of Dolan and More [6] was adopted to compare the new method with the four existing CGMs. For each method, a fraction $P(\tau)$ of the problems for which the method is within a factor $\tau$ of the best time is plotted as shown in Figures 1 and 2. The vertical axis to the left hand side of the curves gives the percentage of the test problems for which a method is the fastest. The percentage of the problems solved successfully for each method, based on the number of iterations and the CPU time, are as follows: $56.6 \%$ for $N M, 49.1 \%$ for $B A N, 50.9 \%$ for $H S, 41.5 \%$ for $C D$, and $83.0 \%$ for $D L$ methods. 
The figures indicate that, based on the number of iterations and the CPU time, the $N M$ method is the next in performance after the $D L$ method, with the $C D$ method as the least performer. This shows that the new method is ranked second out of five methods, thus competing favourably with the existing methods.

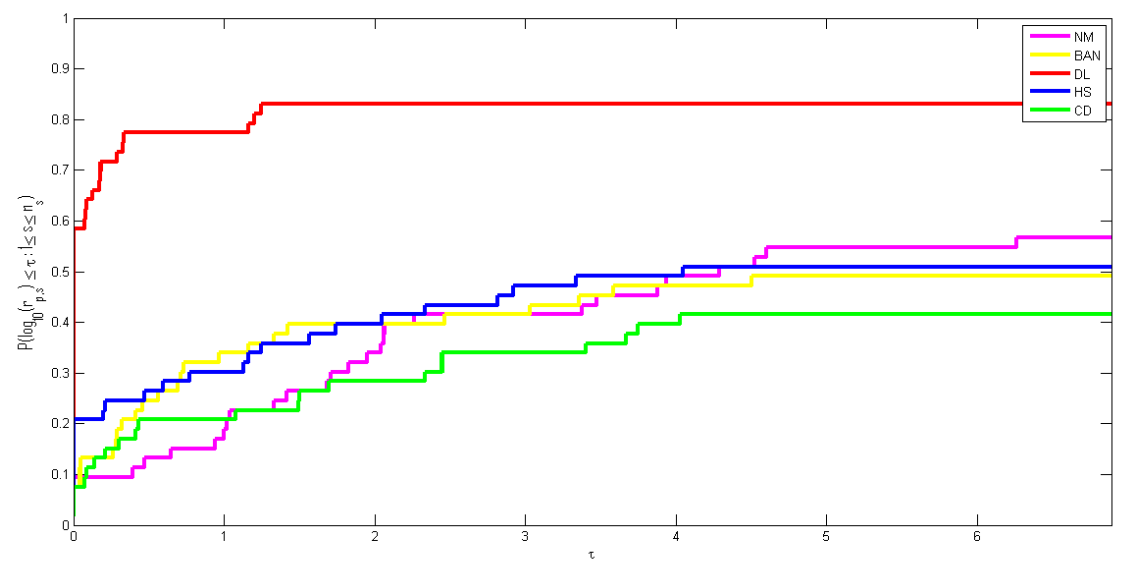

Figure 1: Performance profile for CPU time.

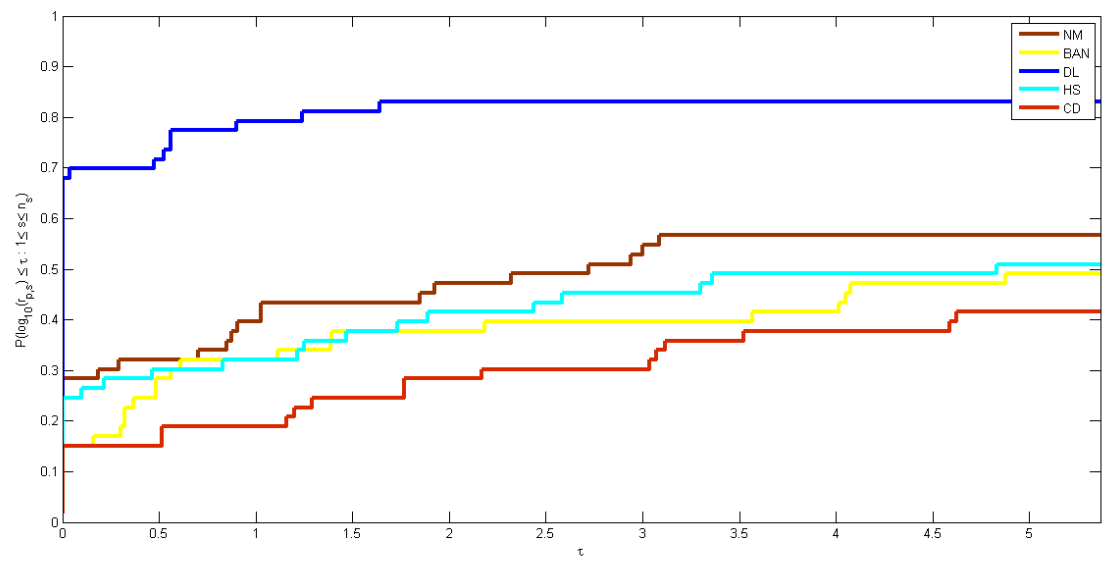

Figure 2: Performance profile for number of iterations. 
Table 1: Numerical Results for NM, BAN and DL Methods

\begin{tabular}{|c|c|c|c|c|c|c|c|c|c|c|}
\hline & & NM & & & BAN & & & DL & & \\
\hline Test problems & Dim & $\left\|g\left(x^{*}\right)\right\|$ & Iter & cpu & $\left\|g\left(x^{*}\right)\right\|$ & Iter & cpu & $\left\|g\left(x^{*}\right)\right\|$ & Iter & cpu \\
\hline \multirow[t]{2}{*}{ Arwhead } & 5000 & $\mathrm{~F}$ & $\mathrm{~F}$ & $\mathrm{~F}$ & $6.12 \mathrm{E}-07$ & 85 & 1.996 & $4.74 \mathrm{E}-07$ & 163 & 3.565 \\
\hline & 10000 & $\mathrm{~F}$ & $\mathrm{~F}$ & $\mathrm{~F}$ & $\mathrm{~F}$ & $\mathrm{~F}$ & $\mathrm{~F}$ & $9.01 \mathrm{E}-07$ & 193 & 7.976 \\
\hline \multirow[t]{2}{*}{ Diagonal 4} & 5000 & $7.30 \mathrm{E}-07$ & 44 & 2.208 & $4.90 \mathrm{E}-07$ & 30 & 0.551 & $\mathrm{~F}$ & $\mathrm{~F}$ & $\mathrm{~F}$ \\
\hline & 10000 & $7.11 \mathrm{E}-07$ & 45 & 2.642 & $6.93 \mathrm{E}-07$ & 30 & 1.306 & $\mathrm{~F}$ & $\mathrm{~F}$ & $\mathrm{~F}$ \\
\hline \multirow[t]{2}{*}{ Diagonal 5} & 5000 & $2.46 \mathrm{E}-07$ & 19 & 0.867 & $2.64 \mathrm{E}-19$ & 7 & 0.095 & $1.97 \mathrm{E}-09$ & 5 & 0.078 \\
\hline & 10000 & $8.09 \mathrm{E}-07$ & 33 & 2.049 & $1.24 \mathrm{E}-19$ & 7 & 0.167 & $2.79 \mathrm{E}-09$ & 5 & 0.139 \\
\hline \multirow[t]{2}{*}{ Extended Beale } & 5000 & $9.01 \mathrm{E}-07$ & 1223 & 137.086 & $\mathrm{~F}$ & $\mathrm{~F}$ & $\mathrm{~F}$ & $\mathrm{~F}$ & $\mathrm{~F}$ & $\mathrm{~F}$ \\
\hline & 10000 & $9.94 \mathrm{E}-07$ & 1243 & 218.033 & $\mathrm{~F}$ & $\mathrm{~F}$ & $\mathrm{~F}$ & $\mathrm{~F}$ & $\mathrm{~F}$ & $\mathrm{~F}$ \\
\hline \multirow[t]{2}{*}{ Extended Block Diagonal } & 5000 & $9.70 \mathrm{E}-07$ & 31 & 1.86 & $\mathrm{~F}$ & $\mathrm{~F}$ & $\mathrm{~F}$ & $9.37 \mathrm{E}-07$ & 43 & 0.969 \\
\hline & 10000 & $5.57 \mathrm{E}-07$ & 41 & 2.913 & $\mathrm{~F}$ & $\mathrm{~F}$ & $\mathrm{~F}$ & $9.03 \mathrm{E}-07$ & 36 & 1.155 \\
\hline \multirow[t]{2}{*}{ Extended Powell } & 5000 & $\mathrm{~F}$ & $\mathrm{~F}$ & $\mathrm{~F}$ & $\mathrm{~F}$ & $\mathrm{~F}$ & $\mathrm{~F}$ & $8.42 \mathrm{E}-07$ & 177 & 10.937 \\
\hline & 10000 & $\mathrm{~F}$ & $\mathrm{~F}$ & $\mathrm{~F}$ & $4.46 \mathrm{E}-07$ & 329 & 22.263 & $\mathrm{~F}$ & $\mathrm{~F}$ & $\mathrm{~F}$ \\
\hline \multirow[t]{2}{*}{ Extended Rosenbrock } & 5000 & $9.84 \mathrm{E}-07$ & 260 & 9.78 & $\mathrm{~F}$ & $\mathrm{~F}$ & $\mathrm{~F}$ & $\mathrm{~F}$ & $\mathrm{~F}$ & $\mathrm{~F}$ \\
\hline & 10000 & $7.91 \mathrm{E}-07$ & 239 & 12.755 & $\mathrm{~F}$ & $\mathrm{~F}$ & $\mathrm{~F}$ & $\mathrm{~F}$ & $\mathrm{~F}$ & $\mathrm{~F}$ \\
\hline \multirow[t]{2}{*}{ Extended Tridiagonal-1 } & 5000 & $\mathrm{~F}$ & $\mathrm{~F}$ & $\mathrm{~F}$ & $\mathrm{~F}$ & $\mathrm{~F}$ & $\mathrm{~F}$ & $9.96 \mathrm{E}-07$ & 48 & 1.042 \\
\hline & 10000 & $\mathrm{~F}$ & $\mathrm{~F}$ & $\mathrm{~F}$ & $\mathrm{~F}$ & $\mathrm{~F}$ & $\mathrm{~F}$ & $9.30 \mathrm{E}-07$ & 50 & 1.244 \\
\hline \multirow[t]{2}{*}{ Generalized Tridiagonal-1 } & 5000 & $8.62 \mathrm{E}-07$ & 155 & 9.917 & $6.17 \mathrm{E}-07$ & 407 & 8.29 & $8.32 \mathrm{E}-07$ & 159 & 3.087 \\
\hline & 10000 & $7.35 \mathrm{E}-07$ & 155 & 12.967 & $6.11 \mathrm{E}-07$ & 406 & 16.111 & $5.36 \mathrm{E}-07$ & 289 & 14.705 \\
\hline \multirow[t]{2}{*}{ Generalized White and Holst } & 5000 & $7.51 \mathrm{E}-07$ & 163 & 10.892 & $\mathrm{~F}$ & $\mathrm{~F}$ & $\mathrm{~F}$ & $9.97 \mathrm{E}-07$ & 80 & 2.566 \\
\hline & 10000 & $7.51 \mathrm{E}-07$ & 163 & 18.046 & $\mathrm{~F}$ & $\mathrm{~F}$ & $\mathrm{~F}$ & $9.97 \mathrm{E}-07$ & 80 & 5.861 \\
\hline \multirow[t]{2}{*}{ Hager } & 5000 & $9.58 \mathrm{E}-07$ & 11 & 1.281 & $\mathrm{~F}$ & $\mathrm{~F}$ & $\mathrm{~F}$ & $1.54 \mathrm{E}-09$ & 9 & 0.307 \\
\hline & 10000 & $1.15 \mathrm{E}-14$ & 13 & 1.533 & $\mathrm{~F}$ & $\mathrm{~F}$ & $\mathrm{~F}$ & $1.46 \mathrm{E}-09$ & 8 & 0.367 \\
\hline \multirow[t]{2}{*}{ Modified Extended Beale } & 5000 & $\mathrm{~F}$ & $\mathrm{~F}$ & $\mathrm{~F}$ & $7.23 \mathrm{E}-07$ & 367 & 17.898 & $1.17 \mathrm{E}-07$ & 31 & 3.236 \\
\hline & 10000 & $\mathrm{~F}$ & $\mathrm{~F}$ & $\mathrm{~F}$ & $7.70 \mathrm{E}-07$ & 1067 & 101.431 & $8.24 \mathrm{E}-07$ & 95 & 15.635 \\
\hline \multirow[t]{2}{*}{ RMODF COSINE } & 5000 & $\mathrm{~F}$ & $\mathrm{~F}$ & $\mathrm{~F}$ & $\mathrm{~F}$ & $\mathrm{~F}$ & $\mathrm{~F}$ & $5.04 \mathrm{E}-07$ & 17 & 0.324 \\
\hline & 10000 & $\mathrm{~F}$ & $\mathrm{~F}$ & $\mathrm{~F}$ & $9.88 \mathrm{E}-07$ & 19 & 0.718 & $7.12 \mathrm{E}-07$ & 17 & 0.485 \\
\hline \multirow[t]{2}{*}{ Staircase1 } & 5000 & $0.00 \mathrm{E}+00$ & 1 & 0.039 & $0.00 \mathrm{E}+00$ & 1 & 0.018 & $0.00 \mathrm{E}+00$ & 1 & 0.012 \\
\hline & 10000 & $0.00 \mathrm{E}+00$ & 1 & 0.032 & $0.00 \mathrm{E}+00$ & 1 & 0.022 & $0.00 \mathrm{E}+00$ & 1 & 0.018 \\
\hline Staircase2 & 5000 & $7.29 \mathrm{E}-08$ & 23 & 0.922 & $\mathrm{~F}$ & $\mathrm{~F}$ & $\mathrm{~F}$ & $0.00 \mathrm{E}+00$ & 3 & 0.038 \\
\hline \multirow[t]{2}{*}{ Diagonal 9} & 5000 & $\mathrm{~F}$ & $\mathrm{~F}$ & $\mathrm{~F}$ & $\mathrm{~F}$ & $\mathrm{~F}$ & $\mathrm{~F}$ & $9.87 \mathrm{E}-07$ & 194 & 2.961 \\
\hline & 10000 & $\mathrm{~F}$ & $\mathrm{~F}$ & $\mathrm{~F}$ & $\mathrm{~F}$ & $\mathrm{~F}$ & $\mathrm{~F}$ & $3.06 \mathrm{E}-07$ & 80 & 2.712 \\
\hline \multirow[t]{2}{*}{ Extended MCCORMCK } & 5000 & $\mathrm{~F}$ & $\mathrm{~F}$ & $\mathrm{~F}$ & $9.33 \mathrm{E}-07$ & 121 & 1.219 & $3.14 \mathrm{E}-07$ & 56 & 0.732 \\
\hline & 10000 & $\mathrm{~F}$ & $\mathrm{~F}$ & $\mathrm{~F}$ & $7.34 \mathrm{E}-07$ & 195 & 2.783 & $5.60 \mathrm{E}-07$ & 43 & 1.245 \\
\hline Extended DENSCHNB & 5000 & $4.87 \mathrm{E}-07$ & 19 & 0.46 & $8.74 \mathrm{E}-07$ & 28 & 0.405 & $3.14 \mathrm{E}-07$ & 28 & 0.332 \\
\hline & 10000 & $6.88 \mathrm{E}-07$ & 19 & 0.558 & $4.69 \mathrm{E}-07$ & 29 & 0.744 & $4.44 \mathrm{E}-07$ & 28 & 0.59 \\
\hline Full Hessian FH3 & 5000 & $3.51 \mathrm{E}-07$ & 20 & 1.7 & $\mathrm{~F}$ & $\mathrm{~F}$ & $\mathrm{~F}$ & $1.34 \mathrm{E}-11$ & 4 & 0.111 \\
\hline & 10000 & $5.92 \mathrm{E}-07$ & 34 & 4.262 & $\mathrm{~F}$ & $\mathrm{~F}$ & $\mathrm{~F}$ & $1.39 \mathrm{E}-11$ & 4 & 0.218 \\
\hline Generalized PSC1 & 5000 & $\mathrm{~F}$ & $\mathrm{~F}$ & $\mathrm{~F}$ & $\mathrm{~F}$ & $\mathrm{~F}$ & $\mathrm{~F}$ & $7.17 \mathrm{E}-07$ & 793 & 12.608 \\
\hline & 10000 & $\mathrm{~F}$ & $\mathrm{~F}$ & $\mathrm{~F}$ & $\mathrm{~F}$ & $\mathrm{~F}$ & $\mathrm{~F}$ & $8.59 \mathrm{E}-07$ & 744 & 18.092 \\
\hline MDF EXPLIN 1 & 5000 & $3.68 \mathrm{E}-07$ & 18 & 1.267 & $8.90 \mathrm{E}-07$ & 147 & 1.246 & $1.13 \mathrm{E}-09$ & 5 & 0.055 \\
\hline & 10000 & $9.54 \mathrm{E}-07$ & 40 & 6.691 & $\mathrm{~F}$ & $\mathrm{~F}$ & $\mathrm{~F}$ & $1.59 \mathrm{E}-09$ & 5 & 0.087 \\
\hline MODF COSINE & 5000 & $\mathrm{~F}$ & $\mathrm{~F}$ & $\mathrm{~F}$ & $\mathrm{~F}$ & $\mathrm{~F}$ & $\mathrm{~F}$ & 7.98E-09 & 3 & 0.042 \\
\hline & 10000 & $\mathrm{~F}$ & $\mathrm{~F}$ & $\mathrm{~F}$ & $\mathrm{~F}$ & $\mathrm{~F}$ & $\mathrm{~F}$ & $1.41 \mathrm{E}-09$ & 3 & 0.066 \\
\hline MODF SINE & 5000 & $1.76 \mathrm{E}-07$ & 1 & 0.048 & $1.76 \mathrm{E}-07$ & 1 & 0.018 & $1.76 \mathrm{E}-07$ & 1 & 0.022 \\
\hline & 10000 & $6.23 \mathrm{E}-08$ & 1 & 0.047 & $6.23 \mathrm{E}-08$ & 1 & 0.031 & $6.23 \mathrm{E}-08$ & 1 & 0.034 \\
\hline NONSCOMP & 5000 & $\mathrm{~F}$ & $\mathrm{~F}$ & $\mathrm{~F}$ & $9.63 \mathrm{E}-07$ & 53 & 0.917 & $1.32 \mathrm{E}-05$ & $\mathrm{~F}$ & $\mathrm{~F}$ \\
\hline & 10000 & $\mathrm{~F}$ & $\mathrm{~F}$ & $\mathrm{~F}$ & $2.58 \mathrm{E}-07$ & 76 & 3.684 & $5.82 \mathrm{E}-06$ & $\mathrm{~F}$ & $\mathrm{~F}$ \\
\hline QUARTC & 5000 & $0.00 \mathrm{E}+00$ & 1 & 0.037 & $0.00 \mathrm{E}+00$ & 1 & 0.018 & $0.00 \mathrm{E}+00$ & 1 & 0.019 \\
\hline & 10000 & $0.00 \mathrm{E}+00$ & 1 & 0.042 & $0.00 \mathrm{E}+00$ & 1 & 0.033 & $0.00 \mathrm{E}+00$ & 1 & 0.034 \\
\hline RMDF GENHUMPS & 5000 & $1.98 \mathrm{E}-07$ & 18 & 1.445 & $\mathrm{~F}$ & $\mathrm{~F}$ & $\mathrm{~F}$ & $9.35 \mathrm{E}-09$ & 10 & 0.139 \\
\hline & 10000 & $\mathrm{~F}$ & $\mathrm{~F}$ & $\mathrm{~F}$ & $\mathrm{~F}$ & $\mathrm{~F}$ & $\mathrm{~F}$ & $1.32 \mathrm{E}-08$ & 10 & 0.236 \\
\hline RMDF SINE & 5000 & $\mathrm{~F}$ & $\mathrm{~F}$ & $\mathrm{~F}$ & $7.76 \mathrm{E}-07$ & 116 & 0.82 & $1.55 \mathrm{E}-08$ & 7 & 0.08 \\
\hline & 10000 & $\mathrm{~F}$ & $\mathrm{~F}$ & $\mathrm{~F}$ & $8.20 \mathrm{E}-07$ & 118 & 1.648 & $2.19 \mathrm{E}-08$ & 7 & 0.137 \\
\hline
\end{tabular}


Table 2: Numerical Results for HS and CD Methods

\begin{tabular}{|c|c|c|c|c|c|c|c|}
\hline & & HS & & & CD & & \\
\hline Test problems & Dim & $\left\|g\left(x^{*}\right)\right\|$ & Iter & cpu & $\left\|g\left(x^{*}\right)\right\|$ & Iter & cpu \\
\hline \multirow[t]{2}{*}{ Arwhead } & 5000 & $2.97 \mathrm{E}-07$ & 69 & 1.595 & $\mathrm{~F}$ & $\mathrm{~F}$ & $\mathrm{~F}$ \\
\hline & 10000 & $8.10 \mathrm{E}-07$ & 62 & 3.357 & $\mathrm{~F}$ & $\mathrm{~F}$ & $\mathrm{~F}$ \\
\hline \multirow[t]{2}{*}{ Diagonal 4} & 5000 & $\mathrm{~F}$ & $\mathrm{~F}$ & $\mathrm{~F}$ & $1.95 \mathrm{E}-07$ & 24 & 0.535 \\
\hline & 10000 & $\mathrm{~F}$ & $\mathrm{~F}$ & $\mathrm{~F}$ & $2.78 \mathrm{E}-07$ & 24 & 0.806 \\
\hline \multirow[t]{2}{*}{ Diagonal 5} & 5000 & $\mathrm{~F}$ & $\mathrm{~F}$ & $\mathrm{~F}$ & $5.20 \mathrm{E}-07$ & 17 & 0.22 \\
\hline & 10000 & $\mathrm{~F}$ & $\mathrm{~F}$ & $\mathrm{~F}$ & $7.36 \mathrm{E}-07$ & 17 & 0.394 \\
\hline \multirow[t]{2}{*}{ Extended Beale } & 5000 & $\mathrm{~F}$ & $\mathrm{~F}$ & $\mathrm{~F}$ & $\mathrm{~F}$ & $\mathrm{~F}$ & $\mathrm{~F}$ \\
\hline & 10000 & $\mathrm{~F}$ & $\mathrm{~F}$ & $\mathrm{~F}$ & $\mathrm{~F}$ & $\mathrm{~F}$ & $\mathrm{~F}$ \\
\hline \multirow[t]{2}{*}{ Extended Block Diagonal } & 5000 & $9.99 \mathrm{E}-07$ & 882 & 16 & $\mathrm{~F}$ & $\mathrm{~F}$ & $\mathrm{~F}$ \\
\hline & 10000 & $\mathrm{~F}$ & $\mathrm{~F}$ & $\mathrm{~F}$ & $\mathrm{~F}$ & $\mathrm{~F}$ & $\mathrm{~F}$ \\
\hline \multirow{2}{*}{ Extended Powell } & 5000 & $\mathrm{~F}$ & $\mathrm{~F}$ & $\mathrm{~F}$ & $\mathrm{~F}$ & $\mathrm{~F}$ & $\mathrm{~F}$ \\
\hline & 10000 & $6.07 \mathrm{E}-07$ & 382 & 25.599 & $\mathrm{~F}$ & $\mathrm{~F}$ & $\mathrm{~F}$ \\
\hline \multirow[t]{2}{*}{ Extended Rosenbrock } & 5000 & $\mathrm{~F}$ & $\mathrm{~F}$ & $\mathrm{~F}$ & $\mathrm{~F}$ & $\mathrm{~F}$ & $\mathrm{~F}$ \\
\hline & 10000 & $\mathrm{~F}$ & $\mathrm{~F}$ & $\mathrm{~F}$ & $\mathrm{~F}$ & $\mathrm{~F}$ & $\mathrm{~F}$ \\
\hline \multirow[t]{2}{*}{ Extended Tridiagonal-1 } & 5000 & $\mathrm{~F}$ & $\mathrm{~F}$ & $\mathrm{~F}$ & $\mathrm{~F}$ & $\mathrm{~F}$ & $\mathrm{~F}$ \\
\hline & 10000 & $\mathrm{~F}$ & $\mathrm{~F}$ & $\mathrm{~F}$ & $\mathrm{~F}$ & $\mathrm{~F}$ & $\mathrm{~F}$ \\
\hline \multirow{2}{*}{ Generalized Tridiagonal-1 } & 5000 & $5.98 \mathrm{E}-07$ & 214 & 4.661 & 3.59 & $\mathrm{~F}$ & $\mathrm{~F}$ \\
\hline & 10000 & $6.51 \mathrm{E}-07$ & 166 & 6.394 & 3.599 & $\mathrm{~F}$ & $\mathrm{~F}$ \\
\hline \multirow{2}{*}{ Generalized White and Holst } & 5000 & $8.43 \mathrm{E}-07$ & 80 & 2.268 & 195.87 & $\mathrm{~F}$ & $\mathrm{~F}$ \\
\hline & 10000 & $8.43 \mathrm{E}-07$ & 80 & 4.668 & 195.87 & $\mathrm{~F}$ & $\mathrm{~F}$ \\
\hline \multirow[t]{2}{*}{ Hager } & 5000 & $\mathrm{~F}$ & $\mathrm{~F}$ & $\mathrm{~F}$ & $8.33 \mathrm{E}-109$ & 22 & 0.647 \\
\hline & 10000 & $\mathrm{~F}$ & $\mathrm{~F}$ & $\mathrm{~F}$ & $\mathrm{~F}$ & $\mathrm{~F}$ & $\mathrm{~F}$ \\
\hline \multirow[t]{2}{*}{ Modified Extended Beale } & 5000 & $6.85 \mathrm{E}-07$ & 115 & 7.095 & $5.56 \mathrm{E}+01$ & $\mathrm{~F}$ & $\mathrm{~F}$ \\
\hline & 10000 & $8.40 \mathrm{E}-07$ & 66 & 12.387 & $7.87 \mathrm{E}+01$ & $\mathrm{~F}$ & $\mathrm{~F}$ \\
\hline \multirow[t]{2}{*}{ RMODF COSINE } & 5000 & $8.60 \mathrm{E}-07$ & 92 & 0.96 & $6.76 \mathrm{E}-07$ & 39 & 1.051 \\
\hline & 10000 & $3.73 \mathrm{E}-07$ & 47 & 2.002 & $7.98 \mathrm{E}-07$ & 38 & 0.562 \\
\hline \multirow[t]{2}{*}{ Staircase1 } & 5000 & $0.00 \mathrm{E}+00$ & 1 & 0.011 & $0.00 \mathrm{E}+00$ & 1 & 0.011 \\
\hline & 10000 & $0.00 \mathrm{E}+00$ & 1 & 0.016 & $0.00 \mathrm{E}+00$ & 1 & 0.017 \\
\hline Staircase2 & 5000 & $\mathrm{~F}$ & $\mathrm{~F}$ & $\mathrm{~F}$ & $7.57 \mathrm{E}-07$ & 26 & 0.402 \\
\hline \multirow[t]{2}{*}{ Diagonal 9} & 5000 & $\mathrm{~F}$ & $\mathrm{~F}$ & $\mathrm{~F}$ & $\mathrm{~F}$ & $\mathrm{~F}$ & $\mathrm{~F}$ \\
\hline & 10000 & $\mathrm{~F}$ & $\mathrm{~F}$ & $\mathrm{~F}$ & $\mathrm{~F}$ & $\mathrm{~F}$ & $\mathrm{~F}$ \\
\hline \multirow[t]{2}{*}{ Extended MCCORMCK } & 5000 & $4.39 \mathrm{E}-07$ & 575 & 5.162 & $\mathrm{~F}$ & $\mathrm{~F}$ & $\mathrm{~F}$ \\
\hline & 10000 & $\mathrm{~F}$ & $\mathrm{~F}$ & $\mathrm{~F}$ & $\mathrm{~F}$ & $\mathrm{~F}$ & $\mathrm{~F}$ \\
\hline \multirow[t]{2}{*}{ Extended DENSCHNB } & 5000 & $5.72 \mathrm{E}-07$ & 19 & 0.568 & $\mathrm{~F}$ & $\mathrm{~F}$ & $\mathrm{~F}$ \\
\hline & 10000 & $9.11 \mathrm{E}-07$ & 63 & 1.323 & $\mathrm{~F}$ & $\mathrm{~F}$ & $\mathrm{~F}$ \\
\hline \multirow[t]{2}{*}{ Full Hessian FH3 } & 5000 & $\mathrm{~F}$ & $\mathrm{~F}$ & $\mathrm{~F}$ & $7.04 \mathrm{E}-07$ & 18 & 0.605 \\
\hline & 10000 & $\mathrm{~F}$ & $\mathrm{~F}$ & $\mathrm{~F}$ & $1.84 \mathrm{E}-07$ & 46 & 2.774 \\
\hline Generalized PSC1 & 5000 & $1.00 \mathrm{E}-06$ & 1408 & 42.258 & 2.9158 & $\mathrm{~F}$ & $\mathrm{~F}$ \\
\hline & 10000 & $9.96 \mathrm{E}-07$ & 1724 & 91.176 & 6.3358 & $\mathrm{~F}$ & $\mathrm{~F}$ \\
\hline MDF EXPLIN 1 & 5000 & $\mathrm{~F}$ & $\mathrm{~F}$ & $\mathrm{~F}$ & $9.40 \mathrm{E}-07$ & 41 & 0.3 \\
\hline & 10000 & $\mathrm{~F}$ & $\mathrm{~F}$ & $\mathrm{~F}$ & $8.87 \mathrm{E}-07$ & 42 & 0.44 \\
\hline MODF COSINE & 5000 & $4.41 \mathrm{E}-03$ & $\mathrm{~F}$ & $\mathrm{~F}$ & $8.26 \mathrm{E}-07$ & 72 & 0.685 \\
\hline & 10000 & $3.12 \mathrm{E}-03$ & $\mathrm{~F}$ & $\mathrm{~F}$ & $9.94 \mathrm{E}-07$ & 74 & 0.886 \\
\hline MODF SINE & 5000 & $1.76 \mathrm{E}-07$ & 1 & 0.018 & $1.76 \mathrm{E}-07$ & 1 & 0.024 \\
\hline & 10000 & $6.23 \mathrm{E}-08$ & 1 & 0.03 & $6.23 \mathrm{E}-08$ & 1 & 0.033 \\
\hline NONSCOMP & 5000 & $8.84 \mathrm{E}-07$ & 126 & 2.051 & $2.45 \mathrm{E}+02$ & $\mathrm{~F}$ & $\mathrm{~F}$ \\
\hline & 10000 & $7.36 \mathrm{E}-07$ & 59 & 1.885 & $4.66 \mathrm{E}+02$ & $\mathrm{~F}$ & $\mathrm{~F}$ \\
\hline QUARTC & 5000 & $0.00 \mathrm{E}+00$ & 1 & 0.025 & $0.00 \mathrm{E}+00$ & 1 & 0.019 \\
\hline & 10000 & $0.00 \mathrm{E}+00$ & 1 & 0.037 & $0.00 \mathrm{E}+00$ & 1 & 0.032 \\
\hline RMDF GENHUMPS & 5000 & $\mathrm{~F}$ & $\mathrm{~F}$ & $\mathrm{~F}$ & $\mathrm{~F}$ & $\mathrm{~F}$ & $\mathrm{~F}$ \\
\hline & 10000 & $\mathrm{~F}$ & $\mathrm{~F}$ & $\mathrm{~F}$ & $\mathrm{~F}$ & $\mathrm{~F}$ & $\mathrm{~F}$ \\
\hline RMDF SINE & 5000 & $4.22 \mathrm{E}-07$ & 69 & 0.81 & $2.64 \mathrm{E}-07$ & 10 & 0.108 \\
\hline & 10000 & $4.57 \mathrm{E}-07$ & 42 & 1.039 & $3.74 \mathrm{E}-07$ & 10 & 0.169 \\
\hline
\end{tabular}




\section{Conclusion}

In this paper, a general form of the DL-BAN CGM was proposed by forming a linear combination of numerator and denominator terms in the two existing classical CGMs. This approach is capable of producing many new methods, which can be obtained by the different arrangements of the coefficients in the DL-BAN update parameter. A new hybrid CGM has been generated from this general form which has been shown to possess a desirable feature, such as the satisfaction of the sufficient descent condition, which is very vital to the global convergence of the method.

A numerical test of the new method in comparison with four existing classical CGMs, confirmed that the new CGM is capable of superior computational performance over a larger number of the existing methods, with respect to the number of iterations and the CPU time. This result is indicative that it is very probable to generate other CGMs from (10) that are computationally optimal in efficiency. Hence, there is a need to explore the DL-BAN CGM for the best possible classical CGM.

\section{References}

[1] N. Andrei, An unconstrained optimization test functions collection, Adv. Model. Optim. 10(1) (2008), 147-161.

[2] O.M. Bamigbola, M. Ali and E. Nwaeze, An efficient and convergent method for unconstrained nonlinear optimization, Proceedings of International Congress of Mathematicians, Hyderabad, India, 2010.

[3] Edwin K.P. Chong and Stanislaw H. Zak, An Introduction to Optimization, 2nd ed., New York: John Wiley Sons, Inc., 2001.

[4] Y.H. Dai and L.Z. Liao, New conjugacy conditions and related nonlinear conjugate gradient methods, Appl. Math. Optim. 43 (2001), 87-101. https://doi.org/10.1007/s002450010019 
[5] S.S. Djordjevic, New hybrid conjugate gradient method as a convex combination of Ls and Fr methods, Acta Math. Sci. 39(1) (2019), 214-228.

https://doi.org/10.1007/s10473-019-0117-6

[6] E.D. Dolan and J.J. More, Benchmarking optimization software with performance profiles, Math. Program. 91 (2002), 201-213.

https://doi.org/10.1007/s101070100263

[7] R. Fletcher and C.M. Reeves, Function minimization by conjugate gradients, The Computer Journal 7 (1964), 149-154. https://doi.org/10.1093/comjnl/7.2.149

[8] R. Fletcher, Practical Methods of Optimization, 2nd ed., Chichester: John Wiley, 1987.

[9] M.R. Hestenes and E. Stiefel, Methods of conjugate gradients for solving linear systems, Journal of Research of the National Bureau of Standards 49 (1952), 409-436. https://doi.org/10.6028/jres.049.044

[10] X. Li and X. Zhao, A hybrid conjugate gradient method for optimization problems, Natural Science 3 (2011), 85-90. https://doi.org/10.4236/ns.2011.31012

[11] O.L. Mangasarian, Nonlinear Programming, New York: McGraw-Hill, 1969.

[12] J.L. Nazareth, Conjugate Gradient Methods, in: Encyclopedia of Optimization (C.A. Floudas and P.M. Pardalos, Editors), Kluwer Academic Publishers, Boston, 1999.

[13] J. Nocedal, Theory of algorithms for unconstrained optimization, Acta Numerica 1 (1992), 199-242. https://doi.org/10.1017/S0962492900002270

[14] I.A. Osinuga and I.O. Olofin, Extended hybrid conjugate gradient method for unconstrained optimization, Journal of Computer Science and its Application 25 (2018), 25-33.

[15] X. Xu and Fy. Kong, New hybrid conjugate gradient methods with the generalized Wolfe line search, SpringerPlus 5 (2016), Article number: 881. https://doi.org/10.1186/s40064-016-2522-9 
This is an open access article distributed under the terms of the Creative Commons Attribution License (http://creativecommons.org/licenses/by/4.0/), which permits unrestricted, use, distribution and reproduction in any medium, or format for any purpose, even commercially provided the work is properly cited. 